\title{
Clinical utility gene card for: Dent disease (Dent-1 and Dent-2)
}

\author{
Michael Ludwig ${ }^{\star, 1}$, Elena Levtchenko ${ }^{2}$ and Arend Bökenkamp ${ }^{3}$ \\ European Journal of Human Genetics (2014) 22, doi:10.1038/ejhg.2014.33; published online 12 March 2014
}

\section{DISEASE CHARACTERISTICS}

1.1 Name of the disease (synonyms)

Dent-1 disease.

$\mathrm{X}$-linked recessive hypophosphatemic rickets, X-linked recessive nephrolithiasis, low-molecular-weight proteinuria with hypercalciuric nephrocalcinosis, Dent's Japan disease, Japanese Dent('s) disease, idiopathic low-molecular-weight proteinuria of Japanese children.

There is genetic heterogeneity: see also Dent-2 disease, which is caused by mutations in the OCRL gene.

\subsection{OMIM\# of the disease}

Dent disease-1 (MIM \#300009).

$\mathrm{X}$-linked recessive hypophosphatemic rickets (MIM \#300554).

X-linked recessive nephrolithiasis with renal failure (MIM \#310468).

Low-molecular-weight proteinuria with hypercalciuric nephrocalcinosis (MIM \#308990).

Dent-2 disease (MIM \#300555).

1.3 Name of the analyzed genes or DNA/chromosome segments CLCN5, Xp11.22-p11.23.

OCRL, Xq25-q26.1.

\subsection{OMIM\# of the gene(s)}

CLCN5, 300008.

OCRL, 300535.

\subsection{Mutational spectrum}

More than 200 Dent-1 disease patients with CLCN5 defects have been described, with disease-causing mutations scattered throughout the gene. ${ }^{1-6}$ The mutational spectrum includes missense $(44 \%)$ and nonsense (26\%) mutations, small deletions/insertions (15\%) and splice defects $(11 \%)$, with a few hotspots, mainly affecting arginine codons. Large insertions/deletions may be detected in around $4 \%$ of the patients. ${ }^{6}$

OCRL mutations in Dent-2 disease patients are not uniformly distributed. Missense mutations are mainly found in exons $8-15$, nonsense or frameshift mutations almost always affect exons 1-7. 6,7

Human wild-type ClC-5/OCRL-1 with their corresponding exon numbering are deposited in GenBank, accession nos. NG_007159.2 and NP_000267.2. CLCN5 and OCRL mutations are included in the
Human Gene Mutation Database (http://www.hgmd.org/) or can be obtained via the Leiden Open Variation Database (http://grenada. lumc.nl/LOVD2/MR/home.php?select_db=CLCN5 or http://www.ncbi. nlm.nih.gov/lovd/home.php?select_db=OCRL). Novel data should be shared through these databases.

\subsection{Analytical methods}

Bi-directional Sanger sequencing of PCR-amplified products comprising the total coding region and the exon-intron boundaries of the CLCN5 gene and, when negative, the OCRL gene.

\subsection{Analytical validation}

Confirmation of the detected mutation at least from a second amplicon, preferentially from an independent biological sample of the index case. Pathogenicity of novel missense variants has to be verified by (i) testing a set of at least 100 chromosomes from normal ethnically matched controls, (ii) considering its deposition in singlenucleotide polymorphism databases and (iii) using in silico prediction methods. The gold standard is analysis of functional consequences of the respective CLCN5/OCRL mutation in Xenopus laevis oocytes or cell models, performed in a few laboratories in the world. Gene transcripts should be analyzed in case of splice mutations.

\subsection{Estimated frequency of the disease}

(Incidence at birth ('birth prevalence') or population prevalence) To date, around 250 families with Dent-1 disease and about 50 patients with Dent-2 disease have been reported. Based on these data and considering the highly variable presentation, disease frequency cannot be estimated.

1.9 If applicable, prevalence in the ethnic group of investigated person

Not applicable.

\subsection{Diagnostic setting}

\begin{tabular}{lll}
\hline & Yes & No \\
A. (Differential) diagnostics & $\Downarrow$ & $\square$ \\
B. Predictive testing & $\Downarrow$ & $\square$ \\
C. Risk assessment in relatives & $\Downarrow$ & $\square$ \\
D. Prenatal & $\bigotimes$ & $\square$ \\
\hline
\end{tabular}

\footnotetext{
${ }^{1}$ Institute for Clinical Chemistry and Clinical Pharmacology, Bonn University Medical Center, Bonn, Germany; ${ }^{2}$ Department of Pediatric Nephrology and Growth and Regeneration, University Hospitals Leuven, Katholieke Universiteit Leuven, Leuven, Belgium; ${ }^{3}$ Department of Pediatric Nephrology, VU University Medical Center, Amsterdam, The Netherlands

*Correspondence: Professor M Ludwig, Institute for Clinical Chemistry and Clinical Pharmacology, Bonn University Medical Center, Sigmund-Freud-Strasse 25, Bonn, Germany. Tel: +49 228 6885418; Fax: +49 228 6885401; E-mail: mludwig@uni-bonn.de
} 
Comment:

The clinical presentation of Dent disease is frequently subtle with the majority of patients being asymptomatic in childhood. Many patients are identified through urine screening for hematuria and proteinuria, a current method in Japanese school children. ${ }^{1,8}$

Although nephrocalcinosis and hypercalciuria are characteristic findings in Dent disease, only low-molecular-weight proteinuria is uniformly present in all patients. ${ }^{9,10}$

Additional features observed in patients are aminoaciduria (in 40$50 \%$ ), phosphate (in 20-25\%) and potassium wasting (in 5-15\%), glycosuria (in 10-20\%), renal tubular acidosis (in 3-5\%) and renal failure in adulthood. ${ }^{9}$ Manifestations of Dent disease are highly variable, even within the same family, and there is no genotypephenotype correlation. ${ }^{10}$

Dent-2 disease is caused by mutations in OCRL, the gene previously identified as the cause of the oculo-cerebro-renal syndrome (Lowe syndrome, OMIM 309000). ${ }^{11,12}$ Patients show a renal phenotype comparable to Dent-1 disease, except for a lower prevalence of nephrocalcinosis. ${ }^{9}$ Dent-2 disease may present with additional (mild) extra-renal features of the Lowe syndrome spectrum, for example, peripheral cataracts, mild cognitive and/or mental impairment, stunted growth and elevated serum $\mathrm{CK} / \mathrm{LDH}$ levels. ${ }^{9}$

Prenatal diagnosis is feasible if the familial mutation is known but is of no clinical benefit.

\section{TEST CHARACTERISTICS}

\subsection{Analytical sensitivity}

(proportion of positive tests if the genotype is present)

Close to $100 \%$. The sensitivity of sequence analysis of PCR-amplified products approaches $100 \%$. A lot of variants have been tested functionally, ${ }^{13-16}$ and the pathogenicity of most mutants has been predicted by publically available algorithms. Nonetheless, errors may occur due to allele dropout and mutations outside the coding region in the promoter, polyA-site, enhancers or intronic variants may be missed.

\subsection{Analytical specificity}

(proportion of negative tests if the genotype is not present)

Nearly $100 \%$. In rare cases, variants may erroneously be interpreted as pathogenic.

\subsection{Clinical sensitivity}

\section{(proportion of positive tests if the disease is present)}

Mutations in CLCN5 (Dent-1 disease; 60\%) and OCRL (Dent-2 disease; $15 \%$ ) account for $75 \%$ of cases with a phenotype of Dent disease. Sensitivity around $75 \%$.

\subsection{Clinical specificity}

(proportion of negative tests if the disease is not present)

The clinical specificity can be dependent on variable factors, such as age or family history. In such cases, a general statement should be given, even if a quantification can only be made case by case.

Close to $100 \%$.

2.5 Positive clinical predictive value

(life-time risk to develop the disease if test is positive)

$100 \%$. But considerable variability in symptoms.
2.6 Negative clinical predictive value (probability of not developing the disease if the test is negative) Mutation demonstrated in index case from the family: 100\%.

Index case in that family not been tested: Consider the X-linked recessive mode of inheritance. If CLCN5 and OCRL are both normal, the negative predictive value is $75 \%$.

\section{CLINICAL UTILITY}

3.1 (Differential) diagnostics: The person is clinically affected 3.1.1 Can a diagnosis be made other than through a genetic test?

\begin{tabular}{ll}
\hline No & \\
Yes & Clinically \\
& Imaging (ultrasound) \\
& Endoscopy \\
& Biochemistry \\
& Electrophysiology \\
& Other: No tracer uptake on DMSA scan (non-specific)
\end{tabular}

Abbreviation: DMSA, dimercaptosuccinic acid.

\subsubsection{Describe the burden of alternative diagnostic methods to the} patient

The diagnosis of Dent disease is based on a high index of clinical suspicion, as the presenting symptoms are non-specific (proteinuria, kidney stones, renal failure).

The biochemical diagnosis of Dent disease is not invasive (spot urine for low-molecular-weight proteinuria, hypercalciuria and variable presence of other proximal tubular dysfunctions). Nephrocalcinosis and kidney stones can usually be demonstrated by abdominal ultrasound. The amount of total protein excretion may be in the nephrotic range, suggesting a glomerular disease. In this situation, genetic testing can avoid a renal biopsy, which is an invasive and potentially dangerous (bleeding) procedure.

Genetic testing may be crucial in patients presenting with end-stage renal disease, as the urine tests are unreliable in this setting (decrease in calcium excretion, non-specific low-molecular-weight proteinuria from tubulo-interstitial damage).

\subsubsection{How is the cost effectiveness of alternative diagnostic methods} to be judged?

The cost effectiveness of renal ultrasound and biochemical studies is very high.

As low-molecular-weight proteinuria is an obligate finding in Dent disease, this test is of crucial importance and can replace genetic testing in siblings of a genetically proven index case. In this situation, urine biochemistry is much more cost-effective than genetic testing. The presence of other biochemical findings in Dent disease is variable.

3.1.4 Will disease management be influenced by the result of a genetic test?

Yes. In cases with nephrotic range proteinuria, who might undergo a renal biopsy or receive immunosuppressive therapy. ${ }^{17}$

3.2 Predictive Setting: the tested person is clinically unaffected but carries an increased risk based on family history.

3.2.1 Will the result of a genetic test influence lifestyle and prevention?

If genetics test demonstrates the presence of mutated allele, high fluid intake is indicated to prevent renal stone formation, and nephrological follow-up will be initiated. Supplementation of electrolytes or alkali if needed. Potential additional medical interventions, such as 
pharmacological therapy of hypercalciuria and potassium citrate (see 4). Monitoring of kidney function.

3.2.2. Which options in view of lifestyle and prevention does a person at risk have if no genetic test has been done?

Urine could be tested for the presence of low-molecular-weight proteinuria, which is an obligate finding in Dent disease.

3.3 Genetic risk assessment in family members of a diseased person 3.3.1 Does the result of a genetic test resolve the genetic situation in that family?

Yes, if a mutation is demonstrated.

3.3.2 Can a genetic test in the index patient save genetic or other tests in family members?

Yes. If the index case has known mutations, siblings, parents and other family members can be screened for disease by testing urine for lowmolecular-weight proteinuria.

\subsubsection{Does a positive genetic test result in the index patient enable a} predictive test in a family member?

Yes.

\subsection{Prenatal diagnosis}

3.4.1 Does a positive genetic rest result in the index patient enable a prenatal diagnosis?

Yes.

\section{IF APPLICABLE, FURTHER CONSEQUENCES OF TESTING}

Please assume that the result of a genetic test has no immediate medical consequences. Is there any evidence that a genetic test is nevertheless useful for a patient or his/her relatives?

Follow-up is warranted in patients with Dent disease. Potential medical interventions include high fluid intake, thiazide diuretics ${ }^{18}$ and potassium citrate supplementation. ${ }^{19}$ Animal studies suggest a positive effect of citrate suppletion in a Clcn5 knockout mouse model (on kidney function and calcium deposits). ${ }^{19}$ Human data have documented a decrease in hypercalciuria with thiazide diuretics, ${ }^{18}$ yet at the expense of hypokalemia in some patients. The long-term efficacy on the progression of renal failure has not been studied, however.

\section{CONFLICT OF INTEREST}

The authors declare no conflict of interest.

\section{ACKNOWLEDGEMENTS}

This work was supported by EuroGentest2 (Unit 2: 'Genetic testing as part of health care'), a Coordination Action under FP7 (Grant Agreement Number 261469) and the European Society of Human Genetics. EL is supported by the Fund for Scientific Research, Flanders (Belgium) (FWOVlaanderen) (Grant Agreement 1801110N).

1 Lloyd SE, Pearce SH, Fisher SE et al: A common molecular basis for three inherited kidney stone diseases. Nature 1996; 379: 445-449.

2 Lloyd SE, Gunther W, Pearce SH et al: Characterisation of renal chloride channel, CLCN5, mutations in hypercalciuric nephrolithiasis (kidney stones) disorders. Hum Mol Genet 1997; 6: 1233-1239.

3 Carballo-Trujillo I, Garcia-Nieto V, Moya-Angeler FJ et al: Novel truncating mutations in the CIC-5 chloride channel gene in patients with Dent's disease. Nephrol Dial Transplant 2003; 18: 717-723.

4 Hoopes Jr RR, Raja KM, Koich A et al: Evidence for genetic heterogeneity in Dent's disease. Kidney Int 2004; 65: 1615-1620.

5 Wu F, Reed AA, Williams SE et al: Mutational analysis of CLC-5, cofilin and CLC-4 in patients with Dent's disease. Nephron Physiol 2009; 112: 53-62.

6 Sekine T, Komoda F, Miura K et al: Japanese Dent disease has a wider clinical spectrum than Dent disease in Europe/USA: genetic and clinical studies of 86 unrelated patients with low-molecular-weight proteinuria. Nephrol Dial Transplant 2013; 29: 376-384

7 Böckenhauer D, Bökenkamp A, Nuutinen M et al: Novel OCRL mutations in patients with Dent-2 disease. J Pediatr Genet 2012; 1: 15-23.

8 Akuta N, Lloyd SE, Igarashi T et al: Mutations of CLCN5 in Japanese children with idiopathic low molecular weight proteinuria, hypercalciuria and nephrocalcinosis. Kidney Int 1997; 52: 911-916.

9 Bökenkamp A, Böckenhauer D, Cheong $\mathrm{HI}$ et al: Dent-2 disease: a mild variant of Lowe syndrome. J Pediatr 2009; 155: 94-99.

10 Ludwig $M$, Utsch $B$, Balluch $B$, Fründ $S$, Kuwertz-Bröking $E$, Bökenkamp $A$ : Hypercalciuria in patients with CLCN5 mutation. Pediatr Nephrol 2006; 21: 1241-1250.

11 Hoopes Jr RR, Shrimpton AE, Knohl SJ et al: Dent disease with mutation in OCRL1. Am J Hum Genet 2005; 76: 260-267.

12 Utsch B, Bökenkamp A, Benz MR et al: Novel OCRL1 mutations in patients with the phenotype of Dent disease. Am J Kidney Dis 2006; 48: 942-954.

13 Ludwig M, Doroszewicz J, Seyberth HW et al: Functional evaluation of Dent's diseasecausing mutations: Implications for $\mathrm{ClC}-5$ channel trafficking and internalization. Hum Genet 2005; 117: 228-237.

14 Grand T, Mordasini D, L'Hoste S et al: Novel CLCN5 mutations in patients with Dent's disease result in altered ion currents or impaired exchanger processing. Kidney Int 2009; 76: 999-1005.

15 Smith AJ, Reed AA, Loh NY, Thakker RV, Lippiat JD: Characterization of Dent's disease mutations of CLC-5 reveals a correlation between functional and cell biological consequences and protein structure. Am J Physiol Renal Physiol 2009; 296: F390-F397.

16 Grand T, L'Hoste S, Mordasini D et al: Heterogeneity in the processing of CLCN5 mutants related to Dent disease. Hum Mutat 2011; 32: 476-483.

17 Fervenza LC: A patient with nephrotic-range proteinuria and focal global glomerulosclerosis. Clin J Am Soc Nephrol 2013; 8: 1979-1987.

18 Raya KA, Schurman S, D'Mello RG et al: Responsiveness of hypercalciuria to thiazide in Dent's disease. J Am Soc Nephrol 2002; 13: 2938-2944.

19 Cebotaru V, Kaul S, Devuyst 0 et al: High citrate diet delays progression of renal insufficiency in the CIC-5 knockout mouse model of Dent's disease. Kidney Int 2005; 68: $642-652$ 